Математички Билтен

ISSN 0351-336X

Vol.39 (LXV) No.2

UDC: 519.226

$2015(29-39)$

Скопје, Македонија

\title{
MODELING AND ESTIMATING MULTITYPE BRANCHING PROCESSES WITH NEGATIVE MULTINOMIAL OFFSPRING DISTRIBUTIONS
}

\author{
ANA STANEVA AND VESSELA STOIMENOVA
}

\begin{abstract}
We consider two - type branching stochastic processes with offspring distributions from the negative multinomial distribution family. We introduce the Bayesian inference in two sampling schemes - when the entire family tree is observed and when observations only on the generation sizes are made. Simulational and computational results are presented.
\end{abstract}

\section{INTRODUCTION}

Branching stochastic processes are models of many real phenomena, where a number of objects is observed, which live some (random) time, reproduce (or "branch") according to some probabilistic law, and die out. These object are often called particles, cells or individuals. They can be of multiple types and may have different locations in space. Their evolution and generation may be independent or according to certain probabilistic laws.

The multitype branching processes (the branching processes with several types of particles) become popular after the pioneering works of Kolmogorov and Dmitriev [14] and Kolmogorov and Sevastyanov [15] from 1947 on their formulation and handling in the Markov case. Since then there is an impressive number of work in the area of branching processes theory and applications (see f.e. the books of Asmussen and Herring [1], Athreya and Ney [2], Harris [8], Jagers [10], Sevastyanov [19], Yakovlev and Yanev [24] and others).

Statistical estimation of the process' characteristics like the mean number of offspring, the criticality of the process, the offspring distribution and others, is an important issue in their study. Some of the most resent approaches devoted to the statistical inference for branching processes can be found in González et al. [4]. The work of Jacob [9] gives a comprehensive overview of the theoretical and statistical methods used in epidemiology. The importance of simulation, computing and more flexible statistical procedures can also be traced in González et al.

2010 Mathematics Subject Classification. Primary 60J80; Secondary 62F15, 62F35.

Key words and phrases. Bivariate negative multinomial distributions, Multitype branching processes. 
[6], [4], [5]. Results on the asymptotics of the relative frequancies in multitype branching processes and applications in cell biology can be traced in Yakovlev and Yanev [25], [26] and Yakovlev et. al. [23].

As in other fields of statistics, there are different approaches for estimation - parametric, nonparametric and semiparametric settings. The parametric approach uses the exact offspring distribution in a specified parametric family like the multivariate power series. The bivariate power series distribution family is a natural generalization of the univariate power series distribution family and a subclass of the multivariate power series distribution family. There are many sources concerning the properties and applications of the multivariate power series distributions. Among them we mention the pioneering papers of Khatri [13], Patil [18], Gerstenkorn [3] and the thorough books on discrete multivariate distributions of Johnson et. al. [11] and discrete bivariate distributions of Kocherlakota[16]. The estimation in the multivariate power series family is of interest in itself. In the class of the univariate power series offspring distributions some topics of the parametric estimation are considered in Stoimenova and Yanev [21] and of the robust parametric estimation - in Stoimenova [22]. In the present paper we consider the maximum likelihood estimation for two-type branching processes with negative multinomial offspring distribution ([20]) for both types of particles.

\section{Two Type Branching Processes}

Let us consider a multitype branching processes with two types of particles:

$$
Z(n+1)=\left(Z_{1}(n+1), Z_{2}(n+1)\right),
$$

where $Z(n+1)$ denotes the size of the $(n+1)$-st generation, which may be split in two groups according to the type of the particle. Consequently $Z(n+1)$ is a bivari-

ate vector $\left(Z_{1}(n+1), Z_{2}(n+1)\right)$, where $Z_{k}(n+1)$ is the number of individuals of type $\mathrm{k}$ in the $(n+1)$-st generation.

On the other hand the individuals of type $k$ in the $(n+1)$-st generation are obtained as a sum of the numbers of offspring of type $k$ of the individuals living in the previous $(n-t h)$ generation (the so called "branching property").

$$
Z_{k}(n+1)=\sum_{s=1}^{Z_{1}(n)} X_{1 s}^{k}(n)+\sum_{s=1}^{Z_{2}(n)} X_{2 s}^{k}(n), \quad k=1,2
$$

where $X_{i s}^{k}(n)$ denotes the number of children of type $k$ of the $s$-th particle of type $i$ in the $(n+1)$-st generation.

Let

$$
Z_{k}(n,(i, j))=\sum_{s=1}^{Z_{k}(n)} I_{\left\{X_{k s}(n)=(i, j)\right\}}
$$

be the number of particles in the $n$-th generation with exactly $(i, j)$ offspring. Here $I_{\left\{X_{k s}(n)=(i, j)\right\}}$ is the indicator variable for the event that a particle of type $k$ has $i$ children of type 1 and $j$ children of type 2 . 
The following basic relations hold:

$$
Z_{k}(n)=\sum_{(i, j)} Z_{k}(n,(i, j))
$$

Furthermore, if $Z_{s}^{k}(n+1)$ denotes the number of particles of type $s$ in the $(n+1)$-th generation with father of type $k$, it can be easily seen that

$$
\begin{aligned}
Z_{1}(n+1) & =Z_{1}^{1}(n+1)+Z_{1}^{2}(n+1) \\
& =\sum_{i} i Z_{1}(n,(i, j))+\sum_{i} i Z_{2}(n,(i, j))
\end{aligned}
$$

and

$$
\begin{aligned}
Z_{2}(n+1) & =Z_{2}^{1}(n+1)+Z_{2}^{2}(n+1) \\
& \left.=\sum_{j} j Z_{1}(n,(i, j))+\sum_{j} j Z_{2}(n,(i, j))\right) .
\end{aligned}
$$

Consequently,

$$
Z(n+1)=\left(\sum_{(i, j)} i . Z(n,(i, j)), \sum_{(i, j)} j \cdot Z(n,(i, j))\right) .
$$

Let $p_{i j}^{k}$ denote the probability that an individual of type $k$ has $(i, j)$ offspring.

In the statistical inference of branching processes usually the following three sampling schemes are used:

- One observes the entire family tree up to the N-th generation (the number of offspring of each particle):

$$
\widetilde{\widetilde{J}}(N)=\left\{X_{i s}(n): s=1,2, . ., Z_{i}(n) ; i=1,2 ; n=0, \ldots, N-1 ;\right\}
$$

- Number of particles with $(i, j) \in J_{k}$ offspring is known, where $J_{k}$ is the support of the offspring distribution:

$$
\widetilde{\mathcal{J}}(N)=\left\{Z_{k}(n,(i, j)):(i, j) \in J_{k} ; \quad k=1,2 ; n=0, \ldots, N-1 ;\right\}
$$

- One can observe the generation sizes only:

$$
\mathcal{J}(N)=\{Z(0), \ldots, Z(N)\} .
$$

\section{The NEGAtive MULTINOMiAL OFFSPRING DistribUtion}

The negative multinomial-NMn distribution is first introduced as a notion in the model of the inverse sampling in multitype Bernoulli trials.

In the papers discussing statistical theory of accident, absenteeism and contagion, the NMn distribution was introduced under the name "multivariate negative binomial distribution". Bates and Neyman are first which threated NMn distribution systematically.

The NMn distribution is a multivariate generalization of the negative binomial distribution. In this discrete distribution all of the counts are positively correlated. 
Suppose an experiment that generates $d+1 \geq 2$ possible outcomes $\left\{X_{0}, X_{1}, \ldots, X_{d}\right\}$ each occuring with non-negative probabilities $\left\{p_{0}, p_{1}, \ldots, p_{d}\right\},\left(\sum_{i=0}^{d} p_{i}=1\right)$.

If the experiment is stopped, once $X_{0}$ reaches the predetermined value $M$, then the distribution of $\left(X_{1}, \ldots, X_{d}\right)$ is called negative multinomial distribution $N M\left(M, p_{1}, \ldots, p_{d}\right)$ with p.d.f.

$$
\begin{array}{r}
f_{\mathbf{p}}\left(x_{1}, \ldots, x_{d}\right)=\frac{\Gamma\left(M+\sum_{j=1}^{d} x_{j}\right)}{\Gamma(M) \prod_{j=1}^{d} x_{j} !} \cdot\left(1+\sum_{j=1}^{d} p_{j}\right)^{-M} \prod_{j=1}^{d}\left(\frac{p_{j}}{1+\sum_{j=1}^{d} p_{j}}\right)^{x_{j}} \\
=\frac{\Gamma\left(M+\sum_{j=1}^{d} x_{j}\right)}{\Gamma(M) \prod_{j=1}^{d} x_{j} !} \cdot\left(1-\sum_{j=1}^{d} p_{j}\right)^{M} \prod_{j=1}^{d} p_{j}^{x_{j}}
\end{array}
$$

where

The negative multinomial distribution belongs to the family of the multivariate power series distributions.

\section{The LIKELIHOOd FUNCTION}

In this section we find maximum likelihood estimators (MLE) for the four unknown parameters $p_{i k}$ in a two-type branching process, where the offspring distribution for both types of particles is negative multinomial

$$
p_{(i, j)}^{k}=\frac{\Gamma(M+i+j)}{\Gamma(M) i ! j !} \frac{p_{1 k}^{i} p_{2 k}^{j}}{\left(1+p_{1 k}+p_{2 k}\right)^{M+i+j}} .
$$

Hence we suppose that the parameter $M$ is known and the same for the both types of particles. This situation is possible if a particle of type 1 or 2 gives birth to two types of particles until $M$ failures (unsuccessfull births) occur.

With the sampling scheme $\tilde{\mathcal{J}}(N)$, the likelihood function has the form:

$$
\begin{aligned}
L(\widetilde{\mathcal{J}}(N) \mid \boldsymbol{\theta})= & \prod_{k=1}^{2} \prod_{n=1}^{N-1} \prod_{(i, j)}\left(p_{(i, j)}^{k}\right)^{Z_{k}(n,(i, j))}= \\
= & \prod_{k=1}^{2} \prod_{n=1}^{N-1} \prod_{(i, j)}\left(\frac{\Gamma(M+i+j)}{\Gamma(M) i ! j !} \frac{p_{1 k}{ }^{i} p_{2 k}{ }^{j}}{\left(1+p_{1 k}+p_{2 k}\right)^{M+i+j}}\right)^{Z_{k}(n,(i, j))}= \\
= & \prod_{k=1}^{2} \prod_{n=1}^{N-1}\left(\prod_{(i, j)} \frac{\Gamma(M+i+j)}{\Gamma(M) i ! j !}\right)^{Z_{k}(n,(i, j))} p_{1 k}^{\sum_{i, j} i Z_{k}(n,(i, j))} p_{2 k}^{\sum_{i, j} j} Z_{k}(n,(i, j)) \\
& \times\left(1+p_{1 k}+p_{2 k}\right)^{-\sum_{(i, j)}(M+i+j) Z_{k}(n,(i, j))}= \\
= & \prod_{k=1}^{2} \prod_{n=1}^{N-1} C_{k}(n, M) p_{1 k}^{Z_{1}^{k}(n+1)} p_{2 k}^{Z_{2}^{k}(n+1)} \times
\end{aligned}
$$




$$
\begin{aligned}
& \times\left(1+p_{1 k}+p_{2 k}\right)^{-M Z_{k}(n)-Z_{1}^{k}(n+1)-Z_{2}^{k}(n+1)}= \\
= & \prod_{k=1}^{2} C_{k}(M) p_{1 k}^{n} Z_{1}^{n=1} Z_{1}^{k}(n) \sum_{2 k}^{n=1} Z_{2}^{k}(n) \\
& \times\left(1+p_{1 k}+p_{2 k}\right)^{-M \sum_{n=0}^{N-1} Z_{k}(n)-\sum_{n=1}^{N} Z_{1}^{k}(n)-\sum_{n=1}^{N} Z_{2}^{k}(n)},
\end{aligned}
$$

where $C_{k}(n, M)$ and $C_{k}(M)$ are constants, not related the parameters of interest.

From the conditions $\frac{\partial L}{\partial p_{1 k}}=0, \quad \frac{\partial L}{\partial p_{2 k}}=0$ we find the MLE for the probabilities $p_{1 k}$ and $p_{2 k}$ :

$$
\widehat{p}_{1 k}=\frac{\sum_{n=1}^{N} Z_{1}^{k}(n)}{M \sum_{n=0}^{N-1} Z_{k}(n)}, \quad \widehat{p}_{2 k}=\frac{\sum_{n=1}^{N} Z_{2}^{k}(n)}{M \sum_{n=0}^{N-1} Z_{k}(n)}, \quad k=1,2 .
$$

\section{The Bayesian estimation}

The Bayesian approach to statistical design and analysis is effective and practical alternative to the frequentist one.

Gonzalez et.al. [6] apply the non-parametric Baysian approach to multitype branching processes. In the present work we use the parametric Bayesian approach, applied for the bivariate negative multinomial offspring distribution in multitype branching processes.

We use the form (4.1) of the likelihood function.

For a conjugate prior distribution of the random vector $\boldsymbol{p}_{\boldsymbol{k}}=\left(p_{1 k}, p_{2 k}\right)$ we choose the Inverted Dirichlet distribution $I D i r i c h l e t\left(\alpha_{k}, \beta_{k}, \gamma_{k}\right), \alpha_{k}, \beta_{k}, \gamma_{k}>0$.

Hence the prior distribution is:

$$
\pi_{k}\left(p_{1 k}, p_{2 k}\right)=\frac{\Gamma\left(\alpha_{k}+\beta_{k}+\gamma_{k}\right)}{\Gamma\left(\alpha_{k}\right) \Gamma\left(\alpha_{k}\right) \Gamma\left(\alpha_{k}\right)} \cdot \frac{p_{1 k}^{\alpha_{k}-1} \cdot p_{2 k}^{\beta_{k}-1}}{\left(1+p_{1 k}+p_{2 k}\right)^{\alpha_{k}+\beta_{k}+\gamma_{k}}} .
$$

Since the two types of particles reproduce independently, we make the assumption that the parameters of their offspring distributions are independent too, and the joint prior distribution has the form

$$
\pi(\boldsymbol{p} ; \boldsymbol{\alpha})=\pi_{1}\left(\boldsymbol{p}_{\mathbf{1}} ; \boldsymbol{\alpha}_{\mathbf{1}}\right) . \pi_{2}\left(\boldsymbol{p}_{\mathbf{2}} ; \boldsymbol{\alpha}_{\mathbf{2}}\right)
$$

Hence we obtain the following posterior distribution of the parameters $\boldsymbol{p}$

$$
\begin{gathered}
f(\boldsymbol{p} \mid \tilde{\mathcal{J}})=\frac{\pi(\boldsymbol{p} ; \boldsymbol{\alpha}) \cdot L(\tilde{\mathcal{J}} \mid \boldsymbol{p})}{\int \pi(\boldsymbol{p} ; \boldsymbol{\alpha}) \cdot L(\tilde{\mathcal{J}} \mid \boldsymbol{p}) \mathrm{d} \boldsymbol{p}} \propto \pi(\boldsymbol{p} ; \boldsymbol{\alpha}) \cdot L(\tilde{\mathcal{J}} \mid \boldsymbol{p}) \\
\propto \prod_{k=1}^{2} \frac{\Gamma\left(\alpha_{1 k}+\alpha_{2 k}+\alpha_{3 k}\right)}{\Gamma\left(\alpha_{1 k}\right) \Gamma\left(\alpha_{2 k}\right) \Gamma\left(\alpha_{3 k}\right)} \frac{p_{1 k}^{\sum_{n=1}^{N} Z_{1}^{k}(n)+\alpha_{k}-1} \cdot p_{2 k}^{n=1} Z_{2}^{k}(n)+\beta_{k}-1}{\left(1+p_{1 k}+p_{2 k}\right)} \stackrel{M \sum_{n=0}^{N} Z_{k}(n)+\sum_{n=1}^{N} Z_{1}^{k}(n)+\sum_{n=1}^{N} Z_{2}^{k}(n)+\alpha_{k}+\beta_{k}+\gamma_{k}}{ }
\end{gathered}
$$


Consequently

$$
f\left(\boldsymbol{p}_{\boldsymbol{k}} \mid \tilde{\mathcal{J}}\right) \sim \text { IDirichlet }\left(\sum_{n=1}^{N} Z_{1}^{k}(n)+\alpha_{k}, \sum_{n=1}^{N} Z_{2}^{k}(n)+\beta_{k}, M \sum_{n=0}^{N-1} Z_{k}(n)+\gamma_{k}\right)
$$

\section{The GiBBs SAMPLER}

The next step of Bayesian approach is simulation of parameters to obtain a sample from the posterior distribution of the parameters.

If the entire family tree in the considered model is not observed, the number of particles having a fixed number of children $Z_{k}(n,(i, j)), k=1,2$ is not available. In the considered model we suppose that only the size of the population in each generation $\mathcal{J}(N)=\{Z(0), \ldots, Z(N)\}$ is observed.

We approximate the posterior density $f(\mathbf{p} \mid \tilde{\mathcal{J}})$ using the Gibbs sampler algorithm.

Gibbs sampler, described D.Geman and S.Geman (1984) is a Markov chain Monte Carlo (MCMC) method used to approximate the sampling from an arbitrary distribution. The main idea of the MCMC methods is to generate a Markov chain whose limiting distribution is equal to the desired distribution.

According to Gonzalez et.al.[6], [4], [5], the conditional distribution can be expressed as a product of conditional probabilities

$$
P(\tilde{\mathcal{J}}(N) \mid \mathcal{J}(N), \mathbf{p})=\prod_{n=0}^{N-1} P\left(\left(Z(n,(i, j)),(i, j) \in J_{k} \mid Z(n), Z(n+1), \mathbf{p}\right)\right.
$$

each of them stemming from a multinomial distribution.

In the case of a two-type branching process (6.1) can be written in the form:

$$
\begin{aligned}
P(\mathbf{Z}(\mathbf{n},(i, j)) \mid \mathbf{Z}(\mathbf{n}), \mathbf{Z}(\mathbf{n}+\mathbf{1}), \mathbf{p})= & \\
= & \frac{\prod_{k=1}^{2}\left(Z_{k}(n)\right) ! \prod_{(i, j)} \frac{P\left(X_{k s}(n)=(i, j)\right)^{Z_{k}(n,(i, j))}}{Z_{k}(n,(i, j)) !}}{P(\mathbf{Z}(\mathbf{n}+\mathbf{1}) \mid \mathbf{Z}(\mathbf{n}), \mathbf{p})}
\end{aligned}
$$

The numerator of (6.2) is Multinomial distribution with parameters, which may be used to generate the sample $\left\{Z_{k}(n,(i, j))\right\}$ on the basis of two consecutive generation sizes $Z(n)$ and $Z(n+1), n=0,1, \ldots, N-1$.

Hance, we can obtain a sample from $P(\tilde{\mathcal{J}}(N) \mid \mathcal{J}(N), \mathbf{p})$.

In the considered model the Gibbs sampler algorithm works by the following way:

Step 0: Initialize $l=0$.

Step 1: Generate : 
- $p_{1}^{(0)} \sim \operatorname{IDirichlet}\left(\alpha_{1}, \beta_{1}, \gamma_{1}\right)$

- $p_{2}^{(0)} \sim$ IDirichlet $\left(\alpha_{2}, \beta_{2}, \gamma_{2}\right)$

Step 2: Iterate :

(1) $l=l+1$

(2) Generate: $\tilde{\mathcal{J}}(\mathcal{N})^{(l)}$ using the conditional distribution $f(\widetilde{\mathcal{J}} \mid \mathcal{J}, \mathbf{p})$ on the basis of multinomial distributions.

(3) Generate:

$$
\begin{aligned}
& p_{1}^{(l)} \sim \text { IDirichlet }\left(\sum_{l=1}^{N} Z_{1}^{1}(l)+\alpha_{1}, \sum_{l=1}^{N} Z_{2}^{1}(l)+\beta_{1}, M \sum_{l=0}^{N-1} Z_{1}(l)+\gamma_{1}\right) \\
& p_{2}^{(l)} \sim \text { IDirichlet }\left(\sum_{l=1}^{N} Z_{1}^{2}(l)+\alpha_{2}, \sum_{l=1}^{N} Z_{2}^{2}(l)+\beta_{2}, M \sum_{l=0}^{N-1} Z_{2}(l)+\gamma_{2}\right)
\end{aligned}
$$

Step 3: Go to Step 2.

\section{Simulation}

For the simulations in this article we used $\mathrm{R}$ - the system for statistical computation and graphics.

Let us first observe the sensitivity of the model to the choice of the parameters. Suppose we have a sample of the parameters from the prior distribution. We make a simulation of the two-type branching process with negative multinomial distribution with parameters from the sample. The trajectories of the processes can be seen on the following graphics.

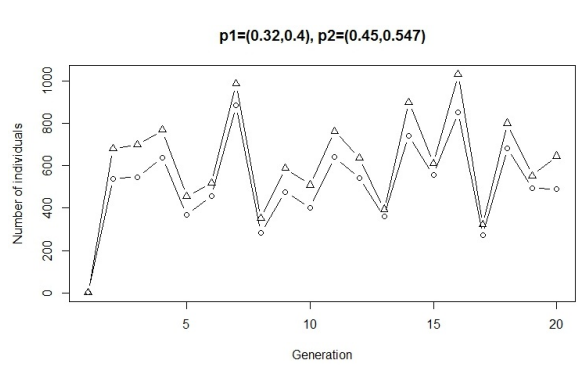

\begin{tabular}{rrrrrrr}
\hline $\mathrm{n}$ & $Z_{1}^{1}(l)$ & $Z_{1}^{2}(l)$ & $Z_{1}(l)$ & $Z_{2}^{1}(l)$ & $Z_{2}^{2}(l)$ & $Z_{2}(l)$ \\
\hline 1 & 0 & 0 & 1 & 0 & 0 & 1 \\
2 & 12 & 526 & 538 & 17 & 663 & 680 \\
3 & 3 & 544 & 547 & 4 & 694 & 698 \\
4 & 4 & 632 & 636 & 5 & 763 & 768 \\
5 & 8 & 359 & 367 & 10 & 445 & 455 \\
6 & 8 & 449 & 457 & 3 & 515 & 518 \\
7 & 8 & 875 & 883 & 5 & 981 & 986 \\
8 & 3 & 279 & 282 & 1 & 350 & 351 \\
9 & 5 & 470 & 475 & 3 & 584 & 587 \\
10 & 4 & 397 & 401 & 2 & 507 & 509 \\
11 & 5 & 635 & 640 & 4 & 758 & 762 \\
12 & 4 & 536 & 540 & 1 & 636 & 637 \\
13 & 7 & 352 & 359 & 8 & 386 & 394 \\
14 & 4 & 738 & 742 & 4 & 895 & 899 \\
15 & 7 & 550 & 557 & 9 & 602 & 611 \\
16 & 14 & 839 & 853 & 16 & 1015 & 1031 \\
17 & 11 & 260 & 271 & 6 & 317 & 323 \\
18 & 3 & 678 & 681 & 4 & 796 & 800 \\
19 & 3 & 490 & 493 & 0 & 552 & 552 \\
20 & 4 & 485 & 489 & 10 & 635 & 645 \\
\hline
\end{tabular}




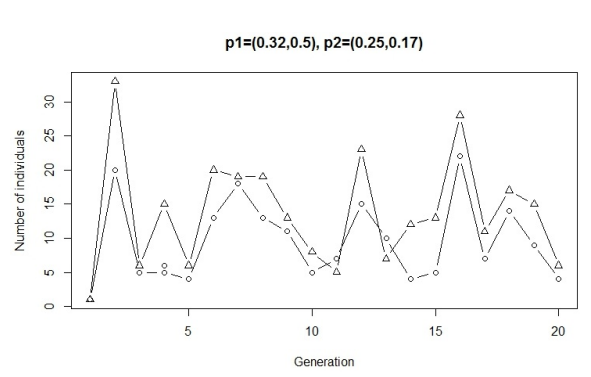

\begin{tabular}{rrrrrrr}
\hline $\mathrm{n}$ & $Z_{1}^{1}(l)$ & $Z_{1}^{2}(l)$ & $Z_{1}(l)$ & $Z_{2}^{1}(l)$ & $Z_{2}^{2}(l)$ & $Z_{2}(l)$ \\
\hline 1 & 0 & 0 & 1 & 0 & 0 & 1 \\
2 & 19 & 1 & 20 & 33 & 0 & 33 \\
3 & 1 & 4 & 5 & 3 & 3 & 6 \\
4 & 4 & 1 & 5 & 11 & 4 & 15 \\
5 & 3 & 1 & 4 & 5 & 1 & 6 \\
6 & 12 & 1 & 13 & 19 & 1 & 20 \\
7 & 13 & 5 & 18 & 19 & 0 & 19 \\
8 & 12 & 1 & 13 & 18 & 1 & 19 \\
9 & 9 & 2 & 11 & 13 & 0 & 13 \\
10 & 3 & 2 & 5 & 7 & 1 & 8 \\
11 & 7 & 0 & 7 & 5 & 0 & 5 \\
12 & 12 & 3 & 15 & 19 & 4 & 23 \\
13 & 6 & 4 & 10 & 7 & 0 & 7 \\
14 & 3 & 1 & 4 & 12 & 0 & 12 \\
15 & 4 & 1 & 5 & 12 & 1 & 13 \\
16 & 18 & 4 & 22 & 27 & 1 & 28 \\
17 & 6 & 1 & 7 & 9 & 2 & 11 \\
18 & 10 & 4 & 14 & 16 & 1 & 17 \\
19 & 6 & 3 & 9 & 15 & 0 & 15 \\
20 & 2 & 2 & 4 & 5 & 1 & 6 \\
\hline
\end{tabular}

On the graphs below the points are the generation sizes (the number of particles of type 1 and the number of particles of type 2), generated via negative multinomial distribution
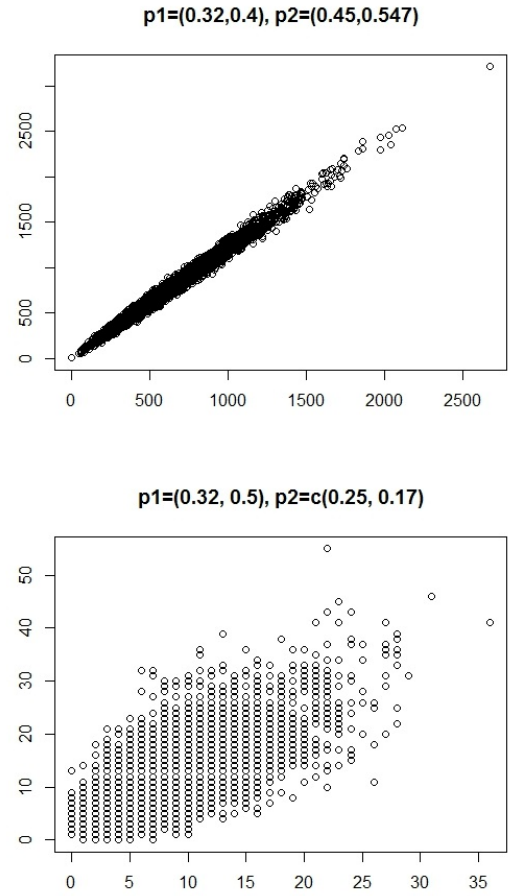


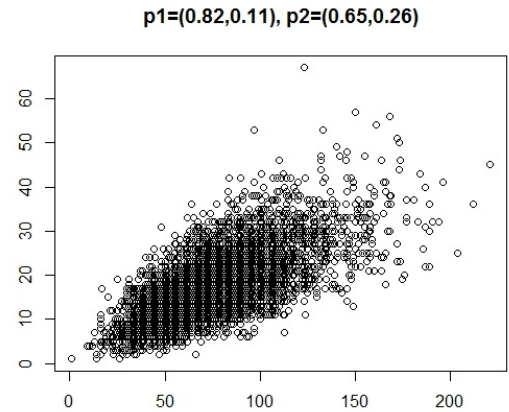

After applying the algorithm, one can obtain the number of children of each type and then calculate the MLE of the parameters. In the table below one can see the estimates after each iteration of the algorithm (we have made 20 iterations). The generated process has negative multionmial offspring distribution with parameters $p_{11}=0.06, p_{21}=0.2, p_{12}=0.3 p_{22}=0.1$. All parameters of the prior distribution are equal to 1 .

\begin{tabular}{rrrrr}
\hline & $\widehat{p}_{11}$ & $\widehat{p}_{21}$ & $\widehat{p}_{12}$ & $\widehat{p}_{22}$ \\
\hline 1 & 0.18367347 & 0.6530612 & 1.1560976 & 0.04878049 \\
2 & 0.10779817 & 0.3698394 & 0.5308353 & 0.02107728 \\
3 & 0.07228916 & 0.2548065 & 0.3302872 & 0.01664491 \\
4 & 0.07465453 & 0.2561510 & 0.3379630 & 0.01978114 \\
5 & 0.06170831 & 0.2135967 & 0.2834414 & 0.01546847 \\
6 & 0.07464444 & 0.2484827 & 0.3261542 & 0.01764234 \\
7 & 0.06788048 & 0.2248303 & 0.2955788 & 0.01623794 \\
8 & 0.06078950 & 0.2053617 & 0.2718778 & 0.01546591 \\
9 & 0.06174485 & 0.2077559 & 0.2739888 & 0.01623596 \\
10 & 0.06767756 & 0.2215780 & 0.2956374 & 0.01816556 \\
11 & 0.06342196 & 0.2096693 & 0.2813661 & 0.01790205 \\
12 & 0.05950262 & 0.1953924 & 0.2590490 & 0.01667850 \\
13 & 0.05892276 & 0.1923781 & 0.2555957 & 0.01615523 \\
14 & 0.06096126 & 0.1985348 & 0.2642744 & 0.01692620 \\
15 & 0.06182473 & 0.2014206 & 0.2678980 & 0.01684366 \\
16 & 0.06085005 & 0.2002387 & 0.2673698 & 0.01701897 \\
17 & 0.05758448 & 0.1900792 & 0.2536528 & 0.01607644 \\
18 & 0.05718179 & 0.1898542 & 0.2520903 & 0.01596791 \\
19 & 0.05763122 & 0.1922251 & 0.2534633 & 0.01616509 \\
20 & 0.05703698 & 0.1907142 & 0.2499405 & 0.01595287 \\
\hline
\end{tabular}

Acknowledgements. This work is partially supported by the National Fund for Scientific Research at the Ministry of Education and Science of Bulgaria, grant NFSI I02/17 and by the European Social Fund through the Human Resource Development Operational Programme under contract BG051PO001-3.3.06-0052 $(2012 / 2014)$ 


\section{REFERENCES}

[1] S. Asmussen, H. Herring, Branching Processes, Birkhauser, Boston, 1983.

[2] K. B. Athreya, P. E. Ney, Branching Processes, Springer-Verlag, Berlin 1972.

[3] T. Gerstenkorn, On multivariate power series distributions, Revue Roumaine de Mathèmatiques Pures et Appliquèes, 26 (1981), 247-266.

[4] M. Gonzalez, C. Gutierrez, R. Martinez, I. del Puerto Controlled branching processes: applications in Biology, Proceedings 59th ISI World Statistics Congress, Hong Kong (Session STS033), (August 25-30, 2013) 1750-1755.

[5] M. Gonzalez, C. Gutierrez, R. Martinez, I. del Puerto Bayesian inference for controlled branching processes through MCMC and ABC methodologies, RACSAM (2013) 107: 459473, DOI 10.1007/s13398-012-0072-8.

[6] M. González, J. Martín, R. Martínez, M. Mota, Non-parametric Bayesian estimation for multitype branching processes through simulation-based methods, Computational statistics \& data analysis, 52, (2008), 1281-1291.

[7] M. González, I. M. del Puerto, R. Martínez, M. Mota, Workshop on Branching Processes and Their Applications, Lecture Notes in Statistics, 197, Springer Verlag, 2010.

[8] T.E. Harris, The Theory of Branching Processes, Springer-Verlag, Berlin, 1963.

[9] C. Jacob, Branching processes: their role in epidemiology, Int. J. Environ. Res. Public Health, 7 (2010), 1186-1204.

[10] P. Jagers, Branching Processes with Biological Applications, Wiley, London, 1975.

[11] N. Johnson, S. Kotz, N. Balakrishtnan, Discrete multivariate distributions, Wiley, London, 1997.

[12] C. G. Khatri, textitOn multivariate contagious distributions, Sankhyia, Series B, 33 (1971), $197-216$.

[13] C. G. Khatri, On Certain Properties of Power-Series Distributions, Biometrika, Vol. 46, No. 3/4 (Dec., 1959), 486-490.

[14] A. N. Kolmogorov, N. A. Dmitriev, Branching random processes, Dokl. Akad. Nauk (Proc. Acad. Sci. USSR), 56 (1947), 7-10, (in Russian).

[15] A. N. Kolmogorov, B. A. Sevastyanov, Calculation of final probabilities of branching random processes, Dokl. Akad. Nauk (Proc. Acad. Sci. USSR), 56, (1947), 783-786, (in Russian).

[16] S. Kocherlakota, K. Kocherlakota, Bivariate discrete distributions, Marcel Dekker Inc., New York, 1992.

[17] T. T. Nguyen, A. K. Gupta, D. M. Nguyen, Y. Wang Characterizations of Negative Multinomial Distributions Based on Conditional Distributions, Metrika, (2007), 66:315-322, DOI 10.1007/s00184-006-0113-4.

[18] G. P. Patil, On multivariate generalized power series distribution and its applications to the multinomial and negative multinomial, Clussical und Contugious Discrete Distributions (Ed. G. P. Patil), Proceedings of International Symposium at McGill University on August 15-20, 183-194, Calcutta: Statistical Publishing Society; Oxford: Pergamon Press, 1965.

[19] B. A. Sevastyanov, Branching Processes, Nauka, Moscow, 1971 (in Russian).

[20] M. Sibuya, I. Yoshimura, R. Shimizu, Negative multinomial distribution, Annals of the Institute of Statistical Mathematics, Vol. 16, Issue 1, (December 1964), 409-426.

[21] V. Stoimenova, N. Yanev, Parametric Estimation in Branching Processes with an Increasing Random Number of Ancestors, Pliska Stud. Math. Bulgar. 17 (2005), 295-312.

[22] V. Stoimenova, Robust Parametric Estimation of Branching Processes with Random Number of Ancestors, Serdica Math. J., 31(3) (2005), 243-262. 
[23] Yakovlev, A.Y., Stoimenova, V.K., Yanev, N.M. Branching Processes as Models of Progenitor Cell Populations and Estimation of the Offspring Distributions, J. American Statistical Assoc., 103 (1998), 1357-1366.

[24] A. Yu. Yakovlev, N. M. Yanev, Transient Processes in Cell Proliferation Kinetics, Springer Verlag, Berlin, 1989.

[25] A. Yu. Yakovlev, N. M. Yanev, Relative frequencies in multitype branching processes, Ann. Appl. Probab., 19, No.1 (2009) 1-14.

[26] A. Yu. Yakovlev, N. M. Yanev, Limiting distributions in multitype branching processes, Stochastic Analysis and Applications, 28, No.6 (2010) 1040-1060.

TeChnical University of Sofia,

8 St.Kliment Ohridski Boulevard, Sofia 1756, Bulgaria,

AND

Department of Probability and Statistics,

Faculty of Mathematics and Informatics, Sofia University,

5 James Bourchier Blvd., 1164, Sofia, Bulgaria

Email address: anastaneva@gmail.com

Department of Probability, Operations Research and Statistics,

Faculty of Mathematics and Informatics, Sofia University,

5 James Bourchier Blvd., 1164, Sofia, Bulgaria

AND

Bulgarian Academy of Sciences, Institute of Mathematics and Informatics,

Acad. Georgi Bonchev Str., Block 8, 1113 Sofia, Bulgaria

Email address: stoimenova@fmi.uni-sofia.bg 